\title{
Efeito da elaboração de mapas conceituais na acurácia diagnóstica de estudantes de medicina
}

\author{
Effect of concept mapping on diagnostic accuracy of medical students
}

Efecto de la construcción de mapas conceptuales en la precisión diagnóstica de estudiantes de medicina

Gláucia Cadar de Freitas Abreu*, Alexandre Sampaio Moura', Eliane Perlatto Moura'.

\section{RESUMO}

Objetivo: Avaliar o impacto da elaboração de mapas conceituais, baseados na fisiopatologia de doenças, sobre a acurácia diagnóstica de estudantes de medicina. Métodos: Estudo experimental com estudantes do $4^{\circ}$ ano de curso de medicina em Belo Horizonte, Minas Gerais. Participaram do estudo 46 alunos, divididos em dois grupos: mapa conceitual (GM) ( $n=24)$ e controle (GC) ( $n=22)$. Na fase de treinamento (fase 1), os alunos resolveram 6 casos clínicos, correspondentes a duas síndromes clínicas. Alunos do GM confeccionaram mapa conceitual e apontaram um diagnóstico final e dois diferenciais. Alunos do GC realizaram atividade distratora. A fase de avaliação (fase 2) foi conduzida uma semana após o treinamento e os participantes foram orientados a listar o diagnóstico mais provável de outros 8 casos clínicos, sem orientação específica. Resultados: Houve uma redução no desempenho dos alunos de ambos os grupos,

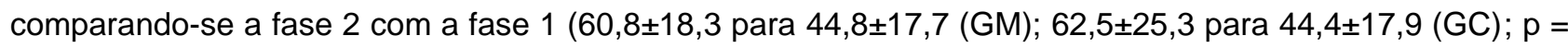
$0,032)$. Não houve associação estatisticamente significativa entre o uso do mapa conceitual e o desempenho dos alunos em nenhuma das duas fases $p=0,577$ ). Conclusão: Não se observou associação entre a elaboração do mapa conceitual orientada para mecanismos fisiopatológicos e acurácia diagnóstica em estudantes de medicina.

Palavras-chave: Educação médica, Materiais de ensino, Aprendizagem por associação, Diagnóstico.

\begin{abstract}
Objective: We aimed to evaluate the impact of concept mapping, based on the pathophysiology of diseases, on the diagnostic accuracy of medical students. Methods: Experimental study with $4^{\text {th }}$ year medical students in Belo Horizonte, Minas Gerais.46 students participated in the study, divided into two groups: concept mapping (GM) $(n=24)$ and control (CG) $(n=22)$. In the training phase (phase 1), students resolved 6 clinical cases, corresponding to two clinical syndromes. GM students made a conceptual map and pointed out a final diagnosis and two differentials. GC students performed a distracting activity. The evaluation phase (phase 2) was conducted one week after the training and the participants were instructed to list the most likely diagnosis of 8 other clinical cases, without specific guidance. Results: We observed a reduction in the students' performance when comparing phase 2 with phase 1 in both groups $(60.8 \pm 18.3$ to $44.8 \pm 17.7$ (GM); $62.5 \pm$ 25.3 to $44.4 \pm 17.9(\mathrm{CG}) ; \mathrm{p}=0.032$ ). There was no association between concept mapping and student performance in either phase $(p=0.577)$. Conclusion: There was no association between the development of the conceptual map oriented to pathophysiological mechanisms and diagnostic accuracy in medical students.
\end{abstract}

Keywords: Medical education, Teaching materials, Association learning, Diagnosis.

1 Universidade José do Rosário Vellano (UNIFENAS), Belo Horizonte - MG.

*E-mail: glaucia.abreu@unifenas.br

SUBMETIDO EM: 12/2020

I ACEITO EM: 12/2020

PUBLICADO EM: 2/2021 


\section{RESUMEN}

Objetivo: Evaluar el impacto de la construcción de mapas conceptuales, basados en la fisiopatología, sobre la precisión diagnóstica de los estudiantes de medicina. Métodos: Estudio experimental con estudiantes de medicina del $4^{\circ}$ ano en Belo Horizonte, Minas Gerais. Participaron 46 estudiantes, divididos en dos grupos: mapa conceptual (GM) $(n=24)$ y control $(C G)(n=22)$. En la fase de entrenamiento (fase 1), los estudiantes resolvieron 6 casos clínicos, correspondientes a dos síndromes. Los estudiantes del GM construyeron un mapa conceptual y señalaron un diagnóstico final y dos diferenciales. Los estudiantes de GC realizaron una actividad distractora. La fase de evaluación (fase 2) se llevó a cabo una semana después y los participantes enumeraran el diagnóstico más probable de 8 casos clínicos, sin orientación específica. Resultados: Hubo una reducción en el desempeño de los estudiantes al comparar la fase 2 con la fase 1 en los dos grupos $(60,8 \pm 18,3$ a 44,8 $\pm 17,7$ (GM); $62,5 \pm 25,3$ a 44,4 $\pm 17,9$ (GC); $p=0,032)$. No hubo asociación estadísticamente significativa entre el uso del mapa conceptual y el desempeño de los estudiantes en ninguna de las fases $(p$ $=0,577)$. Conclusión: No hubo asociación entre la construcción del mapa conceptual y la precisión diagnóstica en estudiantes de medicina.

Palabras clave: Educación médica, Materiales de enseñanza, Aprendizaje por asociación, Diagnóstico.

\section{INTRODUÇÃO}

O raciocínio clínico é utilizado pelo médico para reconhecer adequadamente uma determinada doença como causa dos sinais e sintomas apresentados por um paciente e constitui uma competência clínica fundamental (BOWEN JL, 2006; TEN CO, et al., 2017).

Em medicina, a capacidade de se acertar um diagnóstico diante das evidências clínicas de anamnese, exame físico e exames complementares é chamada de acurácia diagnóstica. De forma geral, é o modo como o médico pensa, organiza ideias e pensamentos, e resgata de maneira automática ou deliberada conceitos adquiridos e aprimorados ao longo de sua formação que, em última instância fazem com que ele acerte 0 diagnóstico e possa oferecer um cuidado melhor ao seu paciente (EVA KW, 2005).

Quando um médico analisa um caso clínico, que aqui será chamado de problema, ele se utiliza de uma rede cognitiva de conhecimentos teóricos básicos - denominado conhecimento biomédico, juntamente com conhecimentos advindos da prática e da experiência - o chamado conhecimento clínico, para resolver da melhor maneira possível este problema. A estruturação do armazenamento do conhecimento médico na memória se modifica ao longo das diferentes fases de aprendizagem. Reestruturar a forma como o conhecimento biomédico e o clínico são armazenados, com o objetivo final de aprimorar o raciocínio clínico e consequentemente apurar a acurácia diagnóstica é um dos desafios do ensino médico (MAMEDE S, 2020).

Com a aplicação extensiva e repetida do conhecimento biomédico adquirido e particularmente através de exposição a problemas clínicos, ocorre uma mudança nas estruturas de conhecimento dos estudantes que começa a tornar-se encapsulado. As suas redes de conhecimento detalhadas, causais e fisiopatológicas, ou seja, analíticas, começam a se reestruturar em categorias de diagnóstico ou modelos mais simplificados, mas de maior nível cognitivo (SCHMIDT HG e RIKERS RM, 2007).

Encapsulamento de conhecimento é um mecanismo de aprendizado que pode ser definido como o agrupamento ou empacotamento de conceitos detalhados de nível cognitivo mais baixo e suas inter-relações, dentro de um número menor de conceitos de nível cognitivo mais alto, com o mesmo poder explicativo. (SCHMIDT HG e RIKERS RM, 2007).

Os scripts mentais das doenças são formas de armazenamento do conhecimento que podem ser acessadas de maneira rápida e fácil para resolver problemas clínicos. Os scripts de doença fazem uso escasso de mecanismos causais, mas são ricos em fatores de risco, sintomas e sinais que caracterizam situações clínicas reais (SCHMIDT HG e RIKERS RM, 2007).

O médico experiente é capaz de acessar uma rede de informações adquiridas, reorganizadas, trabalhadas e encapsuladas através de experiências repetidas da vida real e formar scripts de doenças em sua rede neural 
de conhecimentos para definir diagnósticos com mais rapidez e maior precisão. Desta forma, o raciocínio clínico do expert se dá principalmente de maneira não-analítica, baseado na seleção do script mais apropriado a determinada situação, de maneira rápida e automática. Estudos publicados nas últimas décadas mostram que é possível ensinar, treinar ou aprimorar o raciocínio clínico dos estudantes de medicina nas escolas de médicas com o objetivo final de melhorar a acurácia diagnóstica e consequentemente melhorar o cuidado aos pacientes (EVA KW, 2005).

Uma das estratégias usadas nas escolas com metodologias ativas de ensino médico para ensinar, treinar ou aprimorar o raciocínio médico são os mapas conceituais, que são diagramas de significados, de relações significativas ou de hierarquias conceituais. São propostos como uma estratégia potencialmente facilitadora de uma aprendizagem significativa, pois sua confecção possibilita o aprendizado do aprender, transforma a leitura em pensamento, facilita a identificação de conceitos incorretos (misconceptions), estrutura o raciocínio através de hipóteses, permite a integração de diferentes áreas de conhecimento e promove a integração do conhecimento do grupo (MOREIRA MA, 2013).

O mapeamento de conceitos ajuda os alunos a relacionarem as ciências básicas às clínicas melhorando a compreensão da matéria. Educadores da Universidade de New England College of Osteopathic Medicine desenvolveram uma atividade semanal de mapeamento de conceitos conectando princípios biomédicos a sinais clínicos, sintomas e valores laboratoriais de um caso clínico abrangente o que suscita discussão interdisciplinar, ilustra a integração de conteúdo pelos alunos e aumenta a colaboração do corpo docente entre as disciplinas.

A teoria que está por trás do mapeamento conceitual é a teoria cognitiva de aprendizagem de David Paul Ausubel (1918-2008). A ideia mais importante da teoria de Ausubel pode ser resumida na seguinte proposição de que o fator isolado mais importante que influencia a aprendizagem é aquilo que o aprendiz já sabe. Para Ausubel, aprender significativamente é ampliar e reconfigurar ideias já existentes na estrutura mental e com isso ser capaz de relacionar e acessar novos conteúdos (AUSUBEL DP, et al., 1983)

A teoria da aprendizagem significativa de Ausubel se baseia na prerrogativa que o passado de experiências, emoções e vivências trazidas pelo aluno, junto com a motivação do docente na apresentação de situações ou problemas, favorecem tal aprendizado. Porém, de acordo com ele há duas condições para que a aprendizagem significativa ocorra: o conteúdo a ser ensinado deve ser potencialmente revelador e o estudante precisa estar disposto a relacionar o material de maneira consistente e não arbitrária (AUSUBEL DP, et al., 1983).

A utilização de mapas conceituais é uma técnica flexível para situações e finalidades diferentes, podendo ser usada para uma aula, uma unidade de estudo, um curso ou para o desenvolvimento de todo o programa educacional (CARABETTA JÚNIOR V, 2013).

Os estudos revisados indicam quatro funções principais dos mapas conceituais dentro da educação médica que são: Promoção da aprendizagem significativa, Atuação como recurso adicional de aprendizagem, Feedback, Avaliação da aprendizagem e desempenho (DALEY BJ e TORRE DM, 2010).

A partir da hipótese de que o uso de mapas conceituais pode facilitar o encapsulamento de conceitos e contribuir para o refinamento de scripts mentais, que são fundamentais para a reconstrução do raciocínio clínico, nos propusemos a avaliar a sua utilização baseada nos mecanismos fisiopatológicos das doenças, para o aprimoramento da acurácia diagnóstica em estudantes de medicina.

\section{MÉTODOS}

Foi realizado estudo experimental controlado realizado em duas fases (fase 1- treinamento e fase 2avaliação), com intervalo de uma semana entre elas. A população alvo deste estudo foi constituída por alunos matriculados no 6o período do curso de Medicina da Universidade José do Rosário Vellano. A escolha dos estudantes, nessa etapa da formação, justificou-se por serem alunos que já teriam cursado os blocos Síndromes Cardiológicas e Síndromes Digestórias e, portanto, já apresentados aos temas dos casos clínicos utilizados nas fases do estudo, mas ainda com pouca experiência clínica. Todos os alunos que aceitaram participar foram recrutados como voluntários, e identificados nos dois momentos da aplicação do estudo, 
apenas pelo número da matrícula no curso, para garantir o sigilo das informações e foram classificados em relação ao sexo, idade e gênero. Participaram do estudo 46 alunos, subdivididos em dois grupos. $O$ grupo mapa conceitual (GM) com 24 alunos e o grupo controle (GC) com 22 alunos. A alocação dos alunos nos grupos foi por amostragem aleatória simples.

O estudo foi dividido em duas fases: (1) fase de treinamento e (2) fase de avaliação. Na fase de treinamento, os alunos dos dois grupos resolveram 6 casos clínicos, correspondentes a duas síndromes clínicas (icterícia e dor torácica). Optamos pela escolha destas síndromes clínicas por já terem sido utilizadas e testadas em outro estudo prévio que também visava o aprimoramento do raciocínio clínico e melhoria da acurácia diagnóstica, utilizando outra estratégia educacional (PEIXOTO JM, et al., 2017).

$\mathrm{Na}$ fase 1, os alunos tiveram inicialmente um minuto e meio para ler o caso e listar o diagnóstico mais provável. Após listarem o diagnóstico, os alunos continuaram trabalhando por 8 minutos no mesmo caso clínico, mas de maneiras diferentes. Os alunos do grupo mapa conceitual (GM) foram orientados a confeccionar mapa conceitual, respeitando-se os passos clássicos para sua realização e, então, apontar o diagnóstico final e dois diagnósticos diferenciais.

Os pesquisadores forneceram um arcabouço inicial com uma síntese dos achados clínicos mais relevantes do caso visando otimizar o tempo gasto pelo aluno identificando os conceitos fisiopatológicos mais relevantes para o caso em questão, e a relação entre estes conceitos. O arcabouço inicial não introduziu nenhum conceito novo, se limitando a oferecer, em um outro formato, a informação clínica já presente na descrição inicial do caso clínico.

Os alunos deveriam listar dois diagnósticos e explorar a fisiopatologia de cada um deles, correlacionado com a história clínica apresentada, através da continuação de uma sequência para o mapa conceitual inicialmente fornecido. Os mapas conceituais foram criados à mão com papel e caneta. Os alunos do GC foram orientados a realizar caça-palavras ou jogo dos sete erros e, então, listar o diagnóstico final com dois diagnósticos diferenciais, utilizando o mesmo tempo disponibilizado para o GM.

A duração total da sessão da fase 1 para os dois grupos foi de 90 minutos, incluindo o tempo de orientação dos alunos acerca das atividades a serem realizadas. A definição de tempo foi proposta após realizarmos um projeto piloto para definir um tempo suficiente para a confecção dos mapas sem o risco de cansaço ou dispersão dos alunos.

A fase de avaliação foi conduzida uma semana após a fase de treinamento. Nessa fase, os participantes foram reunidos em uma única sala de aula e convidados a fornecer o diagnóstico de oito casos clínicos, sem orientação sobre a forma de resolução do problema.

Dos oito novos casos clínicos, seis tratam-se de condições clínicas semelhantes aos da fase de treinamento e possuem o mesmo mecanismo fisiopatológico (icterícia e dor torácica), diferentes dos utilizados na fase de treinamento, e dois outros casos comportaram-se como distratores de diagnósticos.

Para cada caso clínico, os estudantes foram orientados a fornecer o diagnóstico principal e dois diagnósticos diferenciais. Todos os diagnósticos fornecidos individualmente pelos estudantes, nas duas fases do estudo, foram listados pelo pesquisador para a atribuição de escore de acerto. A tabela contendo esses diagnósticos foi analisada por três especialistas, que de forma independente pontuaram cada diagnóstico, utilizando uma escala de três pontos (0;0,5 e 1).

A análise da qualidade do mapa foi feita a partir da análise de sua estrutura, atribuindo-se as seguintes notas: 0 ponto: listagem de apenas um ou nenhum diagnóstico e elaboração de apenas um ou nenhum mapa conceitual; 0,5 ponto: listagem de dois diagnósticos, mas elaboração de mapa conceitual para apenas um dos diagnósticos; 1,0 ponto: listagem de dois diagnósticos e inclusão de conceitos e elementos em dois mapas, sendo um para cada diagnóstico.

A acurácia diagnóstica estratificada por síndrome clínica foi realizada somando-se o desempenho do aluno em cada caso correspondente a determinada síndrome. Os resultados de acurácia diagnóstica e conformidade do mapa conceitual (MC) em relação às orientações foram apresentados como percentual do total possível. 
As variáveis quantitativas do estudo foram descritas por meio de Mínimo, Máximo, Mediana, Média e desvio-padrão (d.p) e as variáveis categóricas por meio de proporção. A análise de variância baseada no modelo medidas repetidas (ANOVA) foi utilizada com o objetivo de avaliar a influência da elaboração do mapa conceitual, do tipo de doença e da fase do estudo na acurácia diagnóstica. Neste modelo a acurácia diagnóstica foi analisada como percentual dos pontos obtidos por cada aluno. Apesar da fase de avaliação ter utilizado oito casos, na análise estatística foram considerados apenas os seis casos com diagnósticos que foram treinados na primeira fase, ou seja, três casos para cada síndrome.

A análise de Correlação de Pearson (Teste paramétrico) foi utilizada para avaliar a relação entre a qualidade do mapa conceitual e a acurácia diagnóstica tanto de maneira global quanto estratificado por síndrome clínica. Todos os resultados foram considerados significativos para uma probabilidade de significância inferior a $5 \%(p<0,05)$, tendo, portanto, pelo menos $95 \%$ de confiança nas conclusões apresentadas. O software estatístico utilizado para as análises foi o Statistical Package for the Social Sciences (SPSS) versão 20.0.

\section{RESULTADOS}

Participaram da fase de treinamento 46 alunos, porém, dois alunos do grupo controle (GC) não retornaram para a fase de avaliação e foram excluídos da análise, de modo que os resultados de 44 alunos foram analisados, sendo 24 do grupo mapa conceitual (GM) e 20 do grupo controle (GC). Houve predominância de mulheres em ambos os grupos ( $\mathrm{GM}=62,5 \%$ e $\mathrm{GC}=70 \%$ ), mas não houve diferenças estatísticas em relação ao sexo e idade entre os grupos ( $p=0,839$ e $p=0,663$, respectivamente).

Houve uma redução significativa no desempenho dos alunos comparando-se a fase 2 (avaliação) com a fase 1 (treinamento) $(p=0,032)$, mas não houve associação estatisticamente significativa entre 0 uso do mapa conceitual e o desempenho dos alunos em nenhuma das duas fases $(p=0,577)$.

$\mathrm{Na}$ análise estratificada por síndrome clínica observou-se uma associação entre fase de doença e síndrome clínica $(p<0,001)$, com os alunos apresentando pior acurácia diagnóstica de casos de dor torácica comparando-se a fase de treinamento com a fase de avaliação, enquanto o desempenho para os casos da síndrome ictérica não diferiu entre as fases. Este padrão foi semelhante entre o grupo GM e o $G C(p=0,467)$.

Analisando-se a conformidade dos alunos do grupo GM em elaborar mapas conceituais de acordo com as orientações propostas, observou-se uma baixa conformidade, observando-se uma mediana global de $29,1 \%$, sendo $33,3 \%$ para as síndromes ictéricas e de 16,7\% para dor torácica.

Não houve associação significativa entre a síndrome clínica e a conformidade na realização do mapa conceitual $(p=0,592)$. Não houve correlação entre realizar o mapa conceitual em conformidade com as orientações e a acurácia diagnóstica na fase de avaliação. Na fase de treinamento, houve uma correlação fraca entre acurácia diagnóstica dos casos relacionados à síndrome ictérica e elaboração do mapa em conformidade com as orientações, com um valor de $\boldsymbol{p}$ limítrofe (Tabela 1).

Tabela 1 - Análise de correlação entre a nota obtida pelos alunos no Mapa Conceitual construído e a nota de performance diagnóstica, por tipo de doença

\begin{tabular}{c|c|c|c|c}
\hline & \multicolumn{4}{|c}{ Nota de performance diagnóstica (\%) } \\
\hline Nota no Mapa & \multicolumn{2}{|c|}{ Fase 1 } & $\boldsymbol{2}$ & $\mathbf{r}$ \\
\hline Conceitual (\%) & $\boldsymbol{r}$ & $\mathbf{p}$ & $-0,01$ & 0,963 \\
\hline Ictérica & 0,39 & 0,058 & $-0,10$ & 0,650 \\
\hline Dor torácica & $-0,22$ & 0,298 & \\
\hline
\end{tabular}

Base de dados: Mapa Conceitual $\rightarrow 24$ casos

Nota: $\boldsymbol{r} \rightarrow$ Refere-se ao coeficiente de correlação de Pearson; $\boldsymbol{p} \rightarrow$ Refere-se à probabilidade de significância da Análise de Correlação de Pearson.

Fonte: Abreu GCF, et al., 2018. 


\section{DISCUSSÃO}

Não foi observado efeito do uso dos mapas conceituais (MC) isoladamente, orientado para mecanismos fisiopatológicos das doenças na acurácia diagnóstica de estudantes de medicina do $6^{\circ}$ período.

Apesar dos MC estarem consolidados como uma ferramenta sólida de ensino e aprendizagem na educação médica com estudos mostrando um aprendizado significativo mais duradouro entre alunos que utilizam MC, a sua utilização como intervenção única isolada não resultou em benefício no desenvolvimento do raciocínio clínico (DALEY BJ e TORRE DM, 2010; PUDELKO B, et al., 2012).

Houve uma redução significativa no desempenho dos alunos comparando-se a fase 2 (avaliação) com a fase 1 (treinamento), mas não houve associação estatisticamente significativa entre o uso do mapa conceitual e o desempenho dos alunos em nenhuma das duas fases, ou seja, tanto o grupo GM quanto o GC pioraram o desempenho na fase de avaliação.

Quando analisamos o desempenho dos alunos em relação às síndromes clínicas estudadas, observamos uma piora na acurácia diagnóstica dos casos das síndromes cardiológicas tanto dos alunos do grupo MC quanto do GC quando comparamos as fases de treinamento e a de avaliação. $O$ desempenho para os casos das síndromes ictéricas não diferiu para os dois grupos entre a fase de treinamento e a fase de avaliação.

Talvez a explicação para esta diferença esteja na fisiopatologia das doenças; enquanto a dor torácica envolve múltiplos fatores e pode ter origem em vários sistemas (cardiológico, respiratório, digestório), as síndromes ictéricas, analisadas no estudo tinham origem predominantemente no sistema digestório. Desta forma, o raciocínio para a dor torácica seria mais difícil, justificando um pior desempenho.

Entretanto, estes resultados podem refletir apenas um desbalanceamento da dificuldade dos casos de dor torácica em comparação aos casos da síndrome ictérica, entre as duas fases. Essa piora também foi observada no estudo de Peixoto JM, et al. (2017) que utilizou os mesmos casos clínicos para avaliar o efeito da auto-explicação, o que parece representar uma maior dificuldade, principalmente na resolução dos casos de dor torácica na segunda fase do estudo. Entre as possíveis explicações para a falta do efeito do MC na acurácia diagnóstica, citamos as seguintes:

O tempo para elaboração do mapa conceitual (8 minutos por caso) talvez tenha sido insuficiente para se realizar a tarefa em conformidade com as orientações. Apesar de termos realizado um piloto para verificação deste tempo, percebemos que apenas cerca de um terço dos MC foram realizados exatamente como proposto. Não esperávamos encontrar este tipo de dificuldade pois realizamos o estudo em uma escola na qual os alunos já utilizam o mapeamento de conceitos para resolução de problemas rotineiramente nos grupos tutoriais desde o primeiro período do curso médico.

A construção de mapas em conformidade com as orientações foi ainda menor para os casos de dor torácica, apesar desta diferença entre síndromes não ter alcançado significância estatística. Apesar de alguns estudos mostrarem que os alunos, em geral, apreciam a elaboração de mapas conceituais, outros indicam que os alunos consideram o mapeamento difícil e muito demorado para ser feito, mostrando que a aquisição de habilidades de mapeamento de conceitos pelos alunos é lenta (PUDELKO B, et al., 2012).

O momento de intervenção único e sem feedback pode ter limitado o efeito da elaboração do mapa conceitual na acurácia diagnóstica. A inclusão de feedback imediato após os participantes finalizarem a Fase de Treinamento, talvez possibilitasse a correção de conceitos equivocados (PUDELKO B, et al., 2012; DALEY BJ, 2016).

Uma eventual redução do efeito do uso de $M C$ no grupo experimental pode ter acontecido pelo fato dos alunos (inclusive aqueles do GC) já trabalharem com mapas conceituais. Entretanto os alunos do GC não sabiam a priori que o outro grupo estava utilizando MC para resolução dos casos e o mapeamento conceitual no curso não é utilizado no ensino do raciocínio clínico, tornando esta explicação menos provável.

O pequeno tamanho amostral pode diminuir a força do estudo e dificultar a detecção de um efeito pequeno do mapa conceitual. Entretanto os resultados não sugerem efeito, mesmo pequeno, no grupo experimental, o que parece não respaldar a possibilidade de erro do tipo beta. 
A busca de estratégias de ensino que qualifiquem ou treinem melhor o aluno nos diferentes processos do pensamento, para que os ajudem a vincular a ciência básica com a prática clínica e promover o raciocínio clínico e consequentemente, a melhoria da acurácia diagnóstica é o grande desafio atual do ensino médico. Foi o que nos propusemos a fazer neste estudo. Ajudar estudantes de medicina a tornarem-se diagnosticadores eficazes talvez seja o objetivo mais importante da educação médica (SCHMIDT HG e MAMEDE S, 2015).

A falta de efeito observado em nosso estudo não significa que o MC não funciona para o ensino do raciocínio clínico. Experimentos anteriores, mostram que estratégias educacionais como a reflexão estruturada e a auto-explicação, foram eficazes em melhorar a acurácia diagnóstica e por isso optamos por utilizar um experimento com o mesmo desenho e os mesmos casos clínicos, porém com a utilização de mapas conceituais isoladamente. (PEIXOTO JM, et al., 2017; MAMEDE S, et al., 2014).

Consideramos importante que outros formatos de uso de MC sejam explorados na graduação médica, pois o arcabouço conceitual do uso de MC para a aprendizagem significativa parece muito apropriado para o desenvolvimento do raciocínio clínico. Na educação médica, saber como lidar com grandes quantidades de informações, para que elas sejam efetivamente utilizáveis e aplicáveis ao cotidiano do médico, faz parte do aprendizado. Os estudantes de medicina precisam integrar e conectar várias informações que pertencem a um caso clínico e decidir o melhor caminho a seguir com base nessas informações. A criação de uma base de conhecimento organizada em rede e a possibilidade de se basear nessa rede para tomar decisões clínicas é uma marca da atuação de experts (DALEY BJ e TORRE DM. 2010).

Podem ser explorados outros tipos de instrução para elaboração do mapa conceitual, variações da estrutura inicial de mapa oferecida ao aluno, uso de feedback, além da possibilidade de associação de vários mapas conceituais, de casos clínicos diferentes, a qual proporcionaria a criação de estruturas mais complexas e abrangentes.

\section{CONCLUSÃO}

A abordagem instrucional do MC orientada para mecanismos fisiopatológicos das doenças, com a metodologia usada no presente estudo, não foi capaz de demonstrar melhora na acurácia diagnóstica em estudantes de medicina. O tempo restrito para a elaboração do mapa conceitual, o uso dessa ferramenta isoladamente, fora do contexto do grupo tutorial, no qual os alunos desta escola estão inseridos, assim como a falta de feedback imediato para correção de misconceptions são possíveis explicações para os resultados observados. Novos estudos são necessários para esclarecer o melhor formato de utilização dos MC para desenvolvimento do raciocínio clínico.

\section{REFERÊNCIAS}

1. AUSUBEL DP, et al. Psicología educativa: un punto de vista cognoscitivo. México: Trillas,1983.

2. AZEVEDO AMP, et al. Concept maps as a strategy to assess learning in biochemistry. REB, 2003; (Supl 1):15.

3. BOWEN JL. Educational strategies to promote clinical diagnostic reasoning. NEJM, 2006; 355(21):2217-2225.

4. CARABETTA JÚNIOR V. Using concept maps as a teaching resource for building and interrelating concepts. Rev bras educ méd, 2013; 37(3):441-447.

5. COSTA CRBSF, SIQUEIRA-BATISTA R. As teorias do desenvolvimento moral e o ensino médico: uma reflexão pedagógica centrada na autonomia do educando. Rev bras educ méd, 2004; 28(3):242-50.

6. DALEY BJ, TORRE DM. Concept maps in medical education: an analytical literature review. Med Educ, 2010; 44(5):440-448.

7. DALEY BJ, et al. Using concept maps to create meaningful learning in medical education. MedEdPublish, 2016.

8. DOS SANTOS SL, et al. Mapas conceituais e aprendizagem significativa no ensino de rede de computadores do curso superior de telecomunicações. RENOTE, 2011; 9(2):1-11.

9. EVA KW. What every teacher needs to know about clinical reasoning. Med Educ, 2005; 39(1)98-106.

10. GUL RB, BOMAN JA. Concept mapping: A strategy for teaching and evaluation in nursing education. Nurse Educ Practice, 2006; 6(4):199-206.

11. MAMEDE S, et al. How can students' diagnostic competence benefit most from practice with clinical cases? The effects of structured reflection on future diagnosis of the same and novel diseases. Acad Med, 2014; 89(1):121-127. 
12. MAMEDE S. O que a pesquisa sobre raciocínio clínico tem a dizer a preceptores e professores de áreas clínicas? Sci Med, 2020 ;(Porto Alegre, Online), 37350-37350.

13. MOREIRA MA, MASINI EFS. Aprendizagem significativa: a teoria de aprendizagem de David Ausubel. 2. ed. São Paulo: Centauro Editora, 2006.

14. MOREIRA MA. Aprendizagem significativa, organizadores prévios, mapas conceituais, diagramas $\mathrm{V}$ e unidades de ensino potencialmente significativas. Pontifícia Universidade Católica do Paraná, 2013.

15. NOVAK JD, CANAS AJ. The theory underlying concept maps and how to construct and use them. Technical Report IHMC, 2008.

16. PEIXOTO JM, et al. The effect of self-explanation of pathophysiological mechanisms of diseases on medical students' diagnostic performance. Adv Health Sci Educ, 2017; 22(5):1183-1197.

17. PUDELKO B, et al. Mapping as a learning strategy in health professions education: a critical analysis. Med Educ, 2012; 46(12):1215-1225.

18. SCHMIDT HG, MAMEDE S. How to improve the teaching of clinical reasoning: a narrative review and a proposal. Med Educ, 2015; 49(10):961-973.

19. SCHMIDT HG, RIKERS RM. How expertise develops in medicine: knowledge encapsulation and illness script formation. Med Educ, 2007; 41(12):1133-1139.

20. SPICER DB, et al. Making the Connection: Using Concept Mapping to Bring the Basic Sciences to the Diagnosis. J Am Osteopath Assoc, 2020; 120(8)524-528.

21. TEN CO, et al. Principles and practice of case-based clinical reasoning education: a method for preclinical students. Springer Nature, 2017.

22. WEST DC, et al. Concept mapping assessment in medical education: a comparison of two scoring systems. Med Educ, 2002; 36(9):820-826. 